Corresponding authors: sarah. morton@childrens.harvard.edu; pagrawal@enders.tch.harvard. edu

(C) 2017 Morton et al. This article is distributed under the terms of the Creative Commons Attribution-NonCommercial License, which permits reuse and redistribution, except for commercial purposes, provided that the original author and source are credited.

Ontology terms: brain atrophy; congenital lactic acidosis: elevated brain lactate level by MRS; infantile encephalopathy; lethal infantile mitochondrial myopathy; mitochondrial encephalopathy

Published by Cold Spring Harbor Laboratory Press

doi: $10.1101 / \mathrm{mcs} . a 001560$

\section{AIFM1 mutation presenting with fatal encephalomyopathy and mitochondrial disease in an infant}

\author{
Sarah U. Morton, ${ }^{1,2,3}$ Sanjay P. Prabhu, ${ }^{4}$ Hart G.W. Lidov, ${ }^{5}$ Jiahai Shi, ${ }^{6}$ \\ Irina Anselm, ${ }^{7}$ Catherine A. Brownstein, ${ }^{2,3}$ Matthew N. Bainbridge, ${ }^{8,9}$ \\ Alan H. Beggs, ${ }^{2,3}$ Sara O. Vargas, ${ }^{5}$ and Pankaj B. Agrawal ${ }^{1,2,3}$ \\ ${ }^{1}$ Division of Newborn Medicine, Boston Children's Hospital and Harvard Medical School, Boston, \\ Massachusetts 02115, USA; ${ }^{2}$ Division of Genetics and Genomics, Boston Children's Hospital and Harvard \\ Medical School, Boston, Massachusetts 02115, USA; ${ }^{3}$ The Manton Center for Orphan Disease Research, \\ Boston Children's Hospital and Harvard Medical School, Boston, Massachusetts 02115, USA; ${ }^{4}$ Department of \\ Radiology, Boston Children's Hospital and Harvard Medical School, Boston, Massachusetts 02115, USA; \\ ${ }^{5}$ Department of Pathology, Boston Children's Hospital and Harvard Medical School, Boston, Massachusetts \\ 02115, USA; ${ }^{6}$ Department of Biomedical Sciences, City University of Hong Kong, Hong Kong SAR; \\ ${ }^{7}$ Department of Neurology, Boston Children's Hospital and Harvard Medical School, Boston, Massachusetts \\ 02115, USA; ${ }^{8}$ Rady Children's Institute for Genomic Medicine, San Diego, California 92123, USA; ${ }^{9}$ Codified \\ Genomics LLC, Houston, Texas 77004, USA
}

Abstract Apoptosis-inducing factor mitochondrion-associated 1 (AIFM1), encoded by the gene AIFM1, has roles in electron transport, apoptosis, ferredoxin metabolism, reactive oxygen species generation, and immune system regulation. Here we describe a patient with a novel AIFM1 variant presenting unusually early in life with mitochondrial disease, rapid deterioration, and death. Autopsy, at the age of $4 \mathrm{mo}$, revealed features of mitochondrial encephalopathy, myopathy, and involvement of peripheral nerves with axonal degeneration. In addition, there was microvesicular steatosis in the liver, thymic noninvolution, follicular bronchiolitis, and pulmonary arterial medial hypertrophy. This report adds to the clinical and pathological spectrum of disease related to AIFM1 mutations and provides insights into the role of AIFM1 in cellular function.

\section{INTRODUCTION}

Mitochondrial disease can be due to genetic variants affecting the mitochondrial or nuclear genome. Many well-characterized phenotypes of mitochondrial disease with encephalomyopathy are known, including mitochondrial encephalopathy with lactic acidosis and strokelike episodes (MELAS), Kearns-Sayre syndrome, and Leigh syndrome due to mutations in genes that include SUCLA2, POLG, and VARS2 (Tang et al. 2011; Wong 2012; El-Hattab and Scaglia 2013; Diodato et al. 2015).

Apoptosis-inducing factor mitochondrion-associated 1 (AIFM1) protein is known to have oxidoreductase activity, with roles in electron transport, apoptosis, ferredoxin metabolism, reactive oxygen species generation, and immune system regulation (Susin et al. 1999; Miramar et al. 2001; Pospisilik et al. 2007). It was first identified as a flavoprotein that acts as a mitochondrial effector of apoptosis. Under healthy cellular conditions, AIFM1 is tethered 
COLD SPRING HARBOR Molecular Case Studies
Fatal encephalomyopathy with AIFM1 mutation to the inner mitochondrial matrix where it has a role in ATP production (Susin et al. 1999; Vahsen et al. 2004). AIFM1, the gene encoding AIFM1, is located on the X Chromosome at Xq26.1, and X-linked recessive diseases associated with AIFM1 variants have included mitochondrial encephalomyopathy, Cowchock syndrome (also known as X-linked CharcotMarie-Tooth disease-4), and X-linked deafness (Cowchock et al. 1985; Wang et al. 2006; Ghezzi et al. 2010). In addition, a severe infantile encephalopathy and infantile motor neuron disease has been recently reported (Diodato et al. 2015).

Here we describe a male infant with an X-linked sequence variant in AIFM1 and mitochondrial disease characterized by congenital lactic acidosis, intractable seizures, polyneuropathy, and myopathy. Full postmortem examination, at the age of $4 \mathrm{mo}$, afforded extensive pathologic characterization of this severe phenotype.

\section{RESULTS}

\section{Clinical Presentation and Family History}

The patient was born at 40 week (wk) gestation via spontaneous vaginal delivery to a $27-y r-$ old G3P1 woman following an uneventful pregnancy. His birth weight was at the 82nd percentile for age. Family history included a healthy older sibling, previous maternal first trimester spontaneous abortion, maternal aunt with multiple sclerosis, and a paternal grandmother with seizure disorder. His nursery course was unremarkable and he was discharged home without incident.

At 2 days (d) of life, the patient presented with hypopnea and cyanosis. Venous blood gas after intubation demonstrated significant metabolic acidosis with $\mathrm{pH} 6.67$ and $\mathrm{pCO}_{2}$ of 46 $\mathrm{mmHg}$ (reference range: venous $\mathrm{pH}$ 7.31-7.41, $\mathrm{pCO}_{2}$ 41-51). Glucose was elevated at $174 \mathrm{mg} / \mathrm{dl}$ (reference range: 52-100). Ampicillin, gentamicin, and acyclovir were started for possible infectious etiology. Repeat venous blood gas demonstrated respiratory compensation with $\mathrm{pH} 7.24, \mathrm{pCO}_{2} 24.5 \mathrm{mmHg}$, bicarbonate $10 \mathrm{mmol} / \mathrm{l}$ (reference range: 16$21 \mathrm{mmol} / \mathrm{l})$. Blood gases normalized progressively on the day of admission, and serum bicarbonate levels increased to $15 \mathrm{mmol} / \mathrm{l}$. Complete blood count including lymphocytes, serum electrolytes, cerebrospinal fluid (CSF) cell counts, and CSF glucose at that time were essentially normal for age. Physical exam did not reveal any malformations or dysmorphology. Neurologic exam showed spontaneous movements of all four extremities with positive suck and gag reflexes. Chest X-ray was without infiltrate or other abnormality.

The constellation of early-onset metabolic acidosis without hypoglycemia was concerning for pyruvate dehydrogenase deficiency, so feeds were initially withheld and protein content was slowly advanced in parenteral nutrition. Increasing glucose infusion rates did not worsen the acidosis or lactate level. Biotin, carnitine, thiamine, and coenzyme Q10 supplements were started on day 4. The patient also received sodium citrate/citric acid for his acidosis. Nonmetabolic causes of acidosis such as cardiac shunt, renal bicarbonate wasting, and pulmonary disease were ruled out on further testing. Infectious work up was also negative.

\section{Metabolic and Neurologic Evaluation}

Extensive metabolic evaluation was undertaken. Initial urine organic acids did demonstrate marked increase in lactic acid, pyruvic acid, fumaric acid, and malic acid; subsequent samples demonstrated increases in lactic and pyruvic acid suggestive of a mitochondrial disorder. There were a large amount of ketones and a small dicarboxylic aciduria noted, attributed to the ketosis. Plasma amino acids had transient increases in several branched chain amino acid metabolites, with subsequent testing notable only for being consistent with low protein intake. Urine organic acid profile had a small elevation of propionylglycine 
initially with subsequent testing consistent with carnitine supplementation. Other metabolic testing included normal results for ammonia, pyruvate, free and total carnitine, $\beta$-hydroxybutyrate, acetoacetate, creatine kinase, urine ketones, urine-reducing substances, urine acylglycines, CSF amino acids, and CSF neurotransmitters. 5-Methyltetrahydrofolate, tetrahydrobiopterin, and neopterin profiles were normal. CSF lactate $(6 \mathrm{mmol} / \mathrm{l}$, reference range: 0.9-2.5 $\mathrm{mmol} / \mathrm{l})$ and pyruvate were elevated. Skin fibroblasts demonstrated normal activity of the pyruvate dehydrogenase complex.

MRI/MRA on the second day of life demonstrated underopercularization of the Sylvian fissures and prominence of the bifrontal and bitemporal extra-axial spaces. There was small subdural bleeding over the cerebellum, which was considered birth-related. Myelination pattern was normal for the patient's age. Electroencephalogram (EEG) on the third day of life was suggestive of a moderate diffuse encephalopathy of nonspecific etiology but no electrographic or electro-clinical seizures, and subsequent EEG reading on the fourth day of life was within normal limits for age. Electromyography at $4 \mathrm{wk}$ of age and again at $3 \mathrm{mo}$, demonstrated moderately severe axonal and sensorimotor polyneuropathy. Ophthalmologic evaluation at 8 wk of age was normal without optic nerve anomaly or pigmentary retinopathy. Isoelectric focusing was negative for congenital disorders of glycosylation.

\section{Clinical Genetic Evaluation}

Mitochondrial genome scan had a common synonymous single-nucleotide variant (m.14121 $\mathrm{C}>\mathrm{T}$ ), and no deletions were identified. Gene sequencing detected no pathogenic SURF1 or SCO1 variants, and although multiple variants were found in COX10, POLG, and SCO2, none were known to be pathogenic. Chromosomal microarray revealed a 1.3-Mb paternally inherited duplication at $16 \mathrm{q} 23.3$.

\section{Subsequent Clinical Course}

After initial metabolic testing results were nondiagnostic, formula feedings were reintroduced without difficulty. Cardiac evaluation was normal including normal echocardiograms at $6 \mathrm{~d}$ and 3 months of age. The patient had a second episode of respiratory failure in the setting of severe acidosis at $3 \mathrm{wk}$ of age, requiring intubation for $1 \mathrm{~d}$. His lowest bicarbonate during this second episode was $6 \mathrm{mmol} / \mathrm{l}$ (reference: $17-25 \mathrm{mmol} / \mathrm{l})$, and highest lactate was $21.4 \mathrm{mmol} / \mathrm{l}$ (reference range: $0.5-2.2 \mathrm{mmol} / \mathrm{l}$ ). Dichloroacetate was initiated at $1 \mathrm{mo}$ of age as an investigational drug for congenital lactic acidosis. Following this second episode, head computed tomography (CT) showed new hypodensities in the anteromedial thalami bilaterally. Repeat magnetic resonance imaging (MRI) demonstrated T2 hyperintensity in the bilateral anteromedial thalami with increased signal on diffusion-weighted images, in addition to the bifrontal and bitemporal extra-axial space prominence. After 2 mo in the neonatal intensive care unit (NICU), the patient was stable on room air and was discharged home.

One week after discharge, the patient was readmitted at 2.5 mo of life following a series of cyanotic events associated with seizures. On admission he had lactate levels at his baseline of $3-5 \mathrm{mmol} / \mathrm{l}$, and examination was notable for axial and appendicular hypotonia. He was started on phenobarbital for management of seizures. EEG indicated diffuse encephalopathy, frequent left occipital sharp waves, right occipital sharp waves, and no electrographic seizures. Modified barium swallow study demonstrated silent aspiration of thin liquids and oromotor discoordination with thickened feeds. Repeat MRI demonstrated cerebral atrophy in addition to previous findings. After 1 wk he was again stable on room air, taking thickened feeds by mouth without difficulty and was discharged home.

The patient was again readmitted $2 \mathrm{~d}$ after discharge for an episode of lethargy and cyanosis. EEG at this time demonstrated seizures arising from the right occipital and left parieto-occipital regions, and the absence of a posterior dominant rhythm and slowing in the 
COLD SPRING HARBOR Molecular Case Studies
Fatal encephalomyopathy with AIFM1 mutation

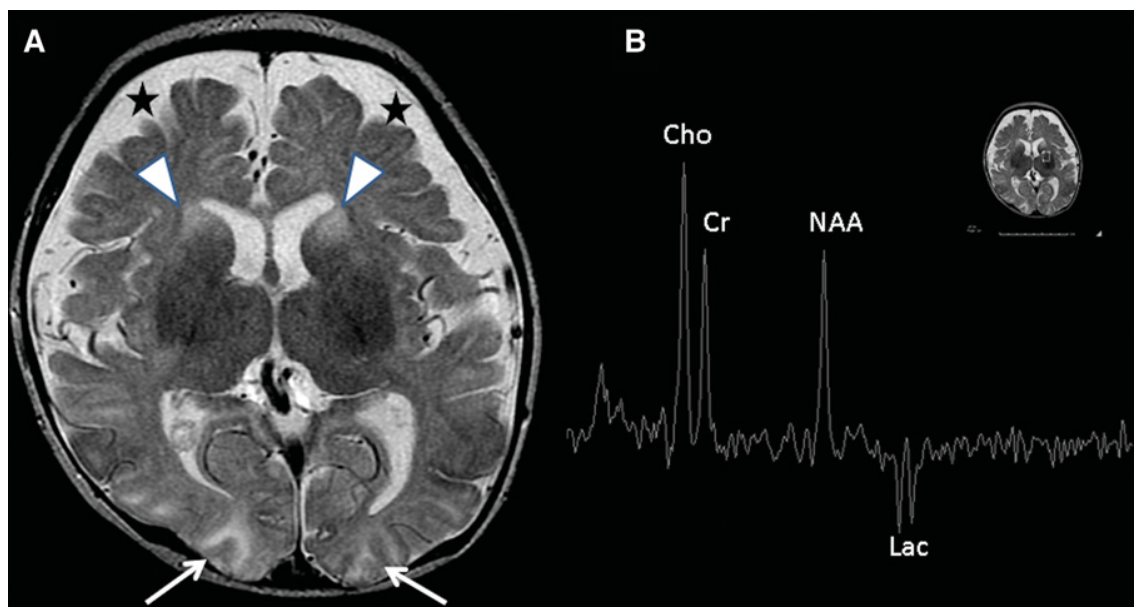

Figure 1. Magnetic resonance imaging (MRI) study demonstrates progression of mitochondrial disease. $(A)$ Restricted diffusion in the basal ganglia, brainstem, and occipital lobe (white arrows) were noted, as well as abnormal T2 signal intensity in the bilateral caudate heads and anterior putamina (arrowheads). Extra-axial spaces were pronounced anteriorly (stars). (B) Inverted lactate doublet is seen at $1.33 \mathrm{ppm}$ on MRI spectroscopy from a voxel placed over the basal ganglia.

posterior quadrants consistent with diffuse encephalopathy. Repeat brain MRI demonstrated new restricted diffusion in the bilateral basal ganglia, brainstem, and bilateral occipital lobes (Fig. 1). There was abnormal T2 signal intensity within the head and body of the caudate nuclei bilaterally and the bilateral anterior putamina (Fig. 1A). A new inverted lactate doublet at $1.33 \mathrm{ppm}$ on single-voxel MR spectroscopy (echo time $144 \mathrm{msec}$ ) was observed within a voxel placed over the left basal ganglia (Fig. 1B). Muscle biopsy during this admission demonstrated decreased activity of electron transport chain complex I and IV, with normal activity of complexes II and III.

During this admission, the patient could not be extubated because of hypoventilation. $\mathrm{He}$ received phenobarbital and gabapentin. The gabapentin was discontinued after a week because of possible gastrointestinal side effects. He had progressive abdominal distension and was unable to tolerate enteral feeds, which was ultimately attributed to progressive mitochondrial dysfunction. There was no significant lactate elevation during this hospitalization. Dichloroacetate was discontinued on admission for concern for worsening neuropathy based on nerve conduction study. Given his poor neurologic prognosis, care was redirected, and he died at the age of $4 \mathrm{mo}$.

\section{Pathology}

\section{Central and Peripheral Nervous System}

Grossly, the brain was of generally normal configuration, weighing $570 \mathrm{~g}$ (expected for age: $700 \mathrm{~g}$ ), and the posterior fossa contents weighed $61 \mathrm{~g}$ (10th percentile). The gyral pattern appeared coarse, and sulci appeared mildly widened. The superior temporal gyrus was thin bilaterally (Fig. 2A) and the corpus callosum and fornix were thin (Fig. 2B). The internal capsule was firm, suggesting gliosis. The occipital cortices appeared thin, and the ventricular system was mildly enlarged, consistent with ex vacuo atrophy.

Microscopically, the frontal and parietal cortices showed a reasonable population of welldifferentiated neurons and normal lamination, although there was some patchy neuronal loss and subtle vacuolation of upper cortical layers II-III. In contrast, the occipital cortex showed profound neuronal loss (Fig. 2C), gliosis, slight prominence of blood vessels (Fig. 2D), and 

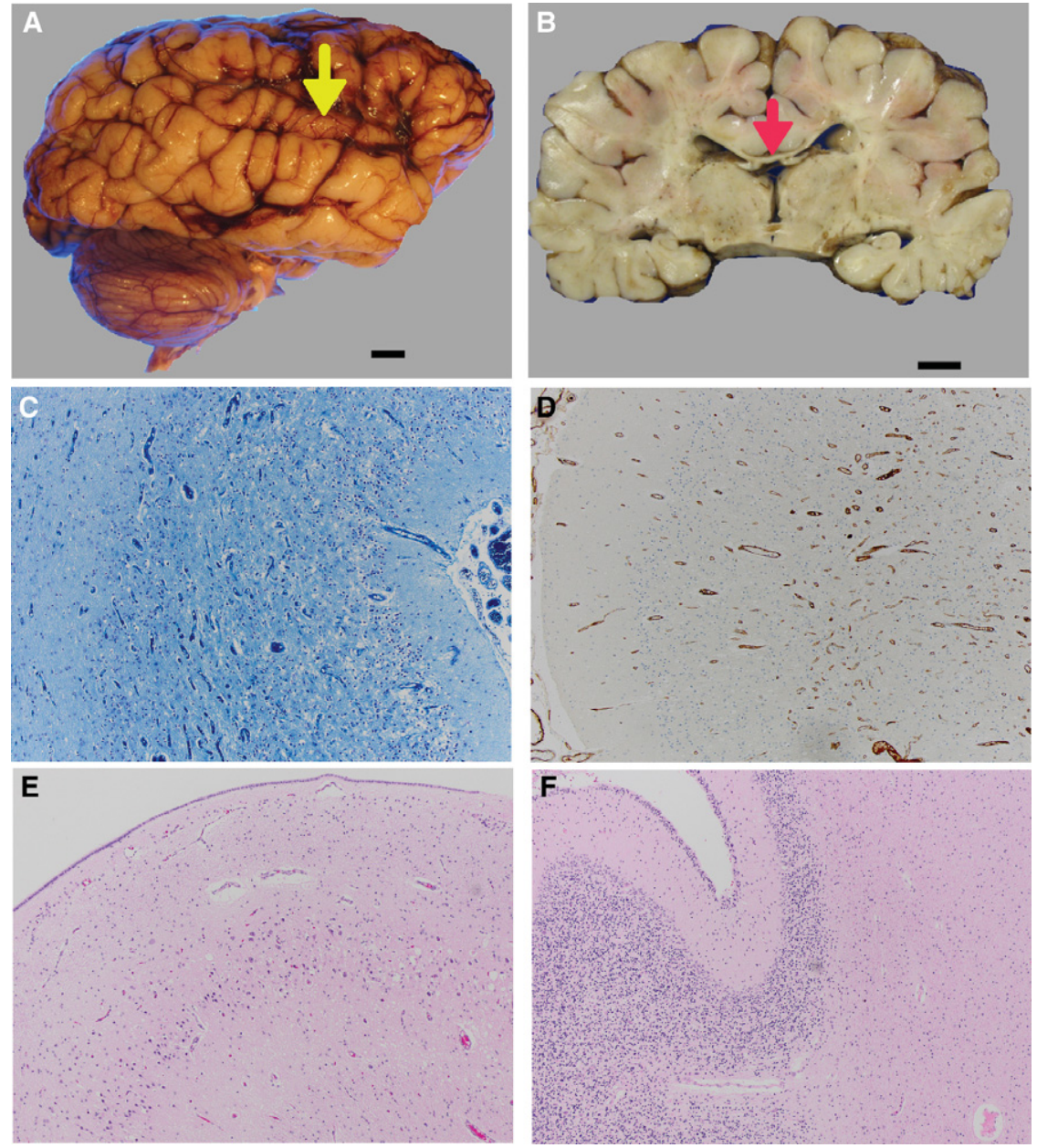

Figure 2. Central nervous system pathological findings on autopsy. (A) The superior temporal gyrus was thin bilaterally (yellow arrowhead). Scale bar, $1 \mathrm{~cm}$. (B) On coronal cut sections, the corpus callosum and fornix were thin (red arrowhead). The ventricular system was mildly enlarged, indicating ex vacuo atrophy. Scale bar, $1 \mathrm{~cm}$. (C) The left occipital cerebral cortex (LFB/CV, 10x) showed spongy neuropil, with almost complete loss of neurons, whereas the underlying white matter was hypomyelinated because of both overlying cortical damage and the patient's young age. (D) Left occipital cerebral cortex (CD34, 10x) showing the prominence and proliferation of small vessels and capillaries in the damaged cerebral cortex. (E) Hippocampal pyramidal cell layer hematoxylin and eosin (H\&E) 10x, showing the marked loss of CA1 pyramidal neurons, and the vacuolation of this layer. $(F)$ Cerebellar cortex, H\&E 10x, showing the depletion of the granular cell layer and widened Virchow-Robin spaces, indicating parenchymal atrophy. The white matter is gliotic (confirmed on glial fibrillary acid protein [GFAP] immunocytochemistry, not shown).

vacuolization of the full thickness of the neocortex with subpial and white matter gliosis. In the hippocampal formation the dentate gyrus and pyramidal cell layer were cytoarchitecturally normally formed. However, the pyramidal cell layer (CA1) was devastated, with marked neuronal loss, vacuolization (Fig. 2E), and scattered hypereosinophilic cells. The cerebellar cortex had the normal layers and a residual external granular layer appropriate for age. However, there was significant patchy loss of Purkinje cells and Bergmann gliosis in the cerebellar hemispheres; these changes became severe, associated with substantial atrophy, in the vermis (Fig. 2F). Glial fibrillary acid protein (GFAP) immunocytochemistry highlighted moderate reactive gliosis in both cortex and white matter. The dentate nucleus was severely 



Figure 3. Peripheral neuromuscular pathological findings. (A) Peripheral nerve, cross section plastic semithin section, toluidine blue, 100x showing generally thin myelin sheaths, likely appropriate for age, and scattered debris-filled profiles of degenerating axons. (B) Peripheral nerve, longitudinal section plastic semithin section, toluidine blue, 100x showing the fusiform profile of a degenerating axon. (C) Electron microscopy of quadriceps muscle demonstrating large, irregularly shaped mitochondria, including one with concentric cisternae (arrow). (D) Quadriceps muscle biopsy (antemortem), Gomori trichrome 100x, showing largely intact fibers with coarse stippling and scattered fibers with subsarcolemmal aggregates corresponding to mitochondria.

depleted of neurons, and there was vacuolation in the caudate and putamen that was less extensive compared with the cortex.

A sample of peripheral nerve showed thin myelination of large axons, appropriate for age, and scattered axonal degeneration, with debris-filled profiles and "myelin ovoids" (Fig. 3A,B).

\section{Muscle Pathology}

Muscle biopsy of the left quadriceps at $8 \mathrm{wk}$ of age demonstrated pyruvate dehydrogenase complex in muscle showed high normal activity. Hematoxylin and eosin (H\&E) stain demonstrated mild variability in fiber size. There was no inflammation, internalized nuclei, degenerating or necrotic fibers, myophagocytosis, or nuclear bags. Trichrome stain revealed normal connective tissue and myofibrillar structure. Periodic acid-Schiff (PAS) stain revealed normal content and appearance of glycogen, and oil red-O stain demonstrated normal content of lipid. Nicotinamide adenine dinucleotide (NADH) histochemical stain revealed the usual finely stippled staining pattern and an unremarkable intermyofibrillar network. ATPase histochemical stains at pH 4.3 and 9.4 showed good distinction of fiber types and a normal distribution of fiber types in a checkerboard pattern. Staining for cytochrome oxidase (COX) was within normal limits. Electron microscopy demonstrated mitochondria that were irregular and bizarrely shaped without inclusions, numerous atrophic fibers, moderate myofiber disarray, and scattered fibers with subsarcolemmal accumulations of glycogen and slightly prominent lipid stores. A mitochondrion with concentric cristae was also noted (Fig. 3C). 

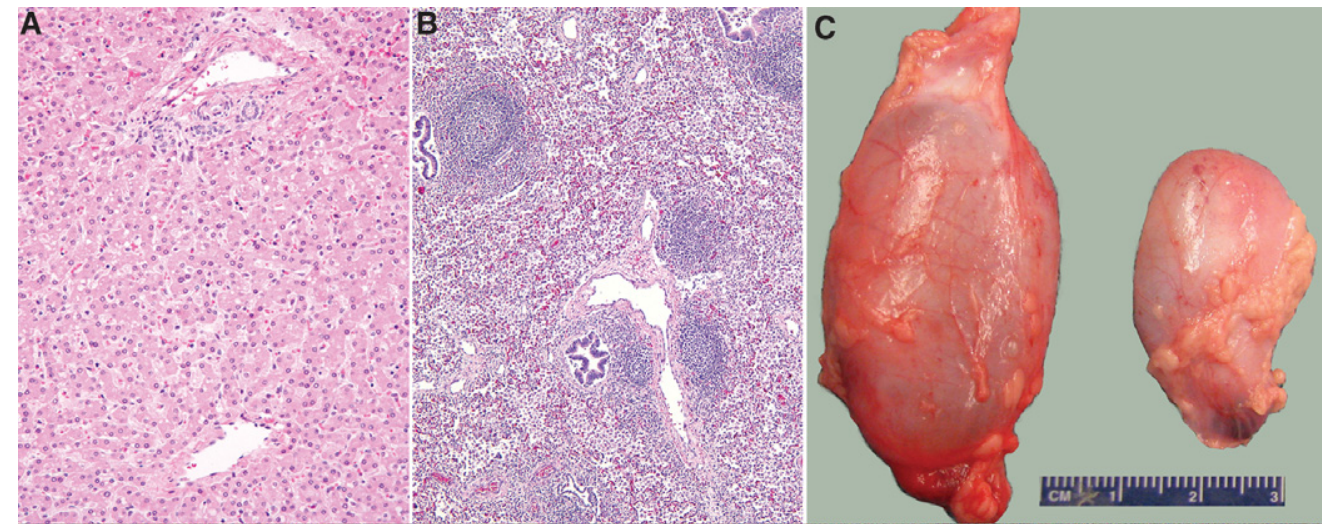

Figure 4. Nonneuromuscular findings on autopsy. (A) Diffuse microvesicular steatosis, characterized by small cytoplasmic vesicles in the majority of hepatocytes (H\&E, original magnification 200x). (B) Follicular bronchiolitis, prominent in the right upper lobe; aggregates of lymphoid cells organized into follicles cuff small airways (H\&E, original magnification 100×). (C) Right (6-cm) and left (3-cm) hydroceles.

Psoas muscle from the autopsy showed increased subsarcolemmal material. A trichrome stain showed a distinct subsarcolemmal accumulation of red-purple material, occasionally approaching frank "ragged red fibers," in the majority of fibers. The toluidine blue semithin sections showed variation in fiber diameter and marked fine cytoplasmic vacuolization, the latter possibly representing postmortem artifact (Fig. 3D). The heart was normal on gross and microscopic examination.

\section{Other Autopsy Findings}

Diffuse microvesicular steatosis was seen in the liver (Fig. 4A). The lungs showed follicular bronchiolitis, most prominent in the right upper lobe (Fig. 4B); in situ hybridization for Epstein-Barr virus early antigen was negative. In addition, small- and medium-sized pulmonary arteries showed moderate concentric medial hypertrophy. The thymus weighed $39 \mathrm{~g}$ and showed a lack of tingible-body macrophages. Hydroceles were present bilaterally, more prominent on the right side (Fig. 4C). The seminal vesicle was distended with abundant globular eosinophilic secretions.

\section{Genomic Analyses}

Whole-exome sequencing was performed on genomic DNA from the proband and his mother. The identified variants in the proband were filtered to include rare variants (mean allele frequency < 0.01) using publicly available Exome Variant Server (http://exac.broadinstitute. org) and 1000 Genomes (http://www.1000genomes.org) databases. Sequencing statistics are available in Table 1. A total of 431 variants were included after the above filtering criteria.

\begin{tabular}{lccccc}
\hline \multicolumn{6}{l}{ Table 1. Sequencing statistics for whole-exome analysis } \\
\hline $\begin{array}{l}\text { Family } \\
\text { members }\end{array}$ & $\begin{array}{c}\text { Total } \\
\text { reads }\end{array}$ & Mapped reads & $\begin{array}{c}\text { Reads on } \\
\text { target }\end{array}$ & $\begin{array}{c}\text { Average } \\
\text { coverage }(x)\end{array}$ & $\begin{array}{c}\text { Target region at 20x } \\
\text { plus coverage }\end{array}$ \\
\hline Proband & $86,633,632$ & $\begin{array}{c}83,531,396 \\
(96.4 \%)\end{array}$ & $\begin{array}{c}51,179,686 \\
(61.3 \%)\end{array}$ & 103 & $95.1 \%$ \\
Mother & $73,649,659$ & $\begin{array}{c}67,263,058 \\
(91.3 \%)\end{array}$ & $\begin{array}{c}42,678,410 \\
(63.5 \%)\end{array}$ & 89 & $94.9 \%$ \\
\hline
\end{tabular}




\begin{tabular}{|c|c|c|c|c|c|c|c|}
\hline Gene & Chromosome & $\begin{array}{l}\text { HGVS DNA } \\
\text { reference }\end{array}$ & $\begin{array}{l}\text { HGVS } \\
\text { protein } \\
\text { reference }\end{array}$ & $\begin{array}{l}\text { Variant } \\
\text { type }\end{array}$ & $\begin{array}{c}\text { Predicted } \\
\text { effect }\end{array}$ & $\mathrm{dbSNP}$ & Genotype \\
\hline AIFM1 & $x$ & $\begin{array}{c}\text { NM_004208. } \\
3: 1436: A\end{array}$ & Gln479 & Substitution & Gln $>$ Arg & 1057516211 & $\begin{array}{l}\text { X-linked } \\
\text { recessive }\end{array}$ \\
\hline
\end{tabular}

Information regarding observed $X$-linked recessive variant in AIFM1.

HGVS, Human Genome Variation Society; dbSNP, Database for Short Genetic Variations.

The variants were first screened for mitochondrial genes, and only one variant in a known mitochondrial gene was identified. The variant was a missense change in AIFM1, an X-linked recessive gene (Table 2). Those 431 mutations were further evaluated for recessive (autosomal/X-linked) changes, and seven genes, including AIFM1, satisfied that criteria. On Sanger sequencing, the proband was confirmed to have a sequence variant in AIFM1 (Fig. 5A), hg19, $X: 129267300 T>C, c .1436 A>G$, which results in the amino acid change $p$. Gln479Arg in exon 13. This variant is not present in the ExAC database and was predicted to be pathogenic by MutationTaster, PolyPhen-2, and SIFT. The altered residue is very well conserved across various vertebrate species (Fig. 5B). The crystal structure of human AIFM1 (PDB code: 4BUR) indicates two NAD and one flavin adenine dinucleotide (FAD) molecules bind to AIFM1. The residue Gln479 is located at a loop forming the binding pocket of

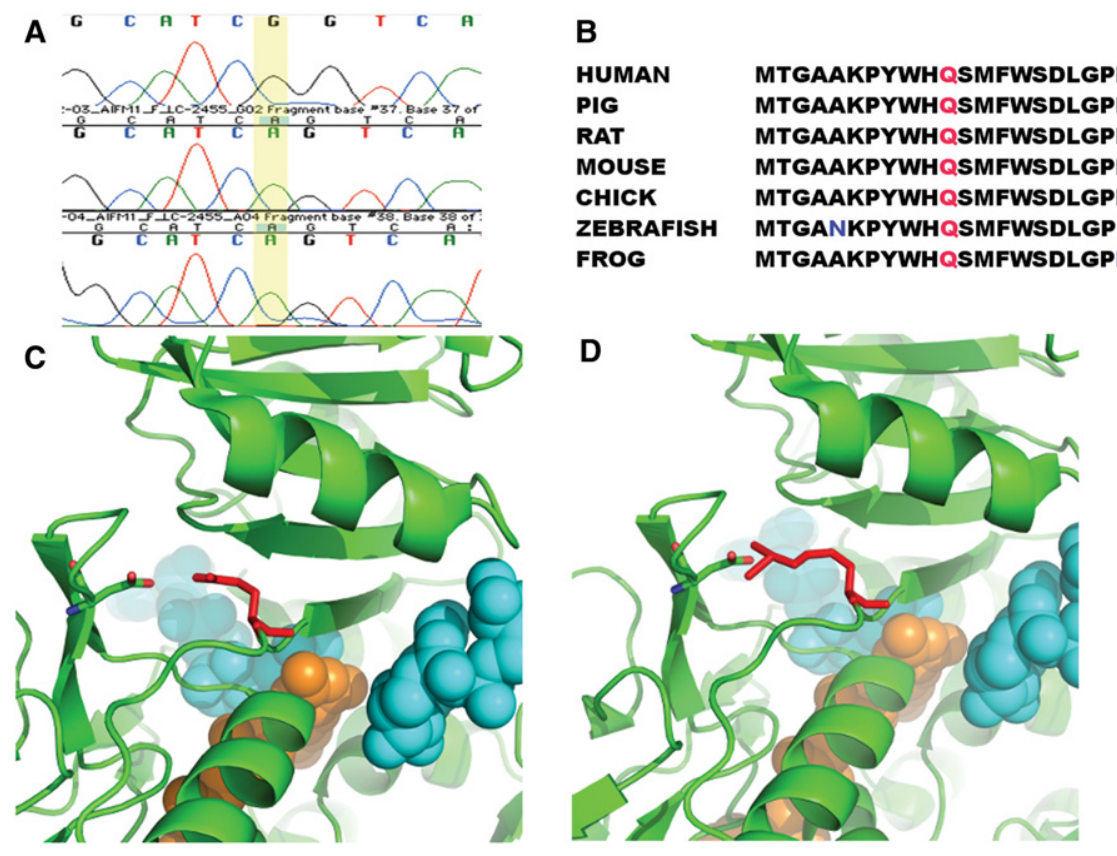

Figure 5. Genetic variation in AIFM1 in affected patient. (A) Sanger sequencing results for affected region of AIFM1. The $A \rightarrow G$ mutation at position 1436 is present in the proband (top row) but not the parents (bottom two rows). (B) Alignment of affected amino acid across vertebrates with residue Gln479 colored in red. Nonconserved amino acids are in blue text. (C) Location of Gln479 variation. One AlFM1 binds to two NAD (colored in cyan) and one FAD (colored in orange). Residue Gln479 (colored in red) is near the binding of the FAD and one NAD. Its amino group interacts with the hydroxyl group of residue Asp443 electrostatically. (D) The NAD and FAD molecules are shown in Sphere. The mutation Gln479Arg does not change the charge status of this residue, but extends the length of the side chain of $\mathrm{G} \ln 479$, which might disrupt the binding pocket of FAD and/or NAD. This change might affect the binding capability of AIFM1 to FAD and/or NAD. 
NAD and FAD. Moreover, Gln479 forms a salt bridge with Asp443, which is located at another NAD and FAD binding loop. The salt bridge links two substrate binding loops, and likely coordinates the dynamic of two loops for substrate binding and enzymatic activities. Strikingly, both Gln479 and Asp443 are not close to other AIFM1 pathogenic mutations, which are around the binding sites of NAD and FAD (Sevrioukova 2016). Mutating Gln479 to Arg479 increases the side chain volume and positive charges significantly. Subsequently this change might alter protein stability or affect the binding capability of AIFM1 to FAD and/or NAD (Fig. 5C,D; Ferreira et al. 2014).

\section{DISCUSSION}

Here we report a case of severe, fatal, early-onset mitochondrial encephalomyopathy in an infant with a novel variant in AIFM1. Previous reports have associated AIFM1 mutations with abnormalities of the nervous system including progressive mitochondrial encephalomyopathy (Ghezzi et al. 2010), X-linked Cowchock syndrome (Rinaldi et al. 2012; Zimoń et al. 2012), slowly progressive mitochondrial disease (Ardissone et al. 2015), auditory neuropathy spectrum disorder (Zong et al. 2015), X-linked congenital ptosis and intellectual disability (Møller et al. 2014), and prenatal ventriculomegaly without lactic acidosis (Table 3; Berger et al. 2011). The Harlequin mouse, a mouse with mutation in Aifm 1 that affects the redox and DNA binding activities of AIFM1, is associated with decreased activity of complex I/ III, neuronal degradation and muscle atrophy (Klein et al. 2002; Joza et al. 2005).

There have been several genotype-phenotype correlations made in previous case reports which both point toward the multiple roles of AIFM1 in cell regulation as well as the pathophysiology of related human conditions. AIFM1 mutations that impair oxidative phosphorylation have presented within the first year of life with severe mitochondrial encephalomyopathy. Alternatively, mutations that affect the redox properties of AIF lead to increased cell death and have been associated with Cowchock syndrome. Most similarly to our case, there were two related male infants with severe mitochondrial encephalomyopathy who had both defects in oxidative phosphorylation as well as increased apoptosis (Ghezzi et al. 2010). Clearly there are also tissue-specific effects, as muscle had more significant respiratory chain dysfunction than skin fibroblasts (Ghezzi et al. 2010; Berger et al. 2011).

There were several notable neuromuscular system findings in our infant on histopathological examination. Brain tissue damage was found to be bilaterally symmetric, and the extent of capillary proliferation and occipital white matter injury was consistent with that found

\begin{tabular}{lll}
\hline \multicolumn{2}{l}{ Table 3. Summary of previously identified human AIFM1 variants } \\
\hline Variant & \multicolumn{1}{c}{ Phenotype } & \multicolumn{1}{c}{ Reference } \\
\hline Arg201del & Severe mitochondrial encephalomyopathy & Ghezzi et al. 2010 \\
Gly338Glu & Early-onset mitochondrial encephalomyopathy & Diodato et al. 2015 \\
Glu493Val & Early-onset axonal sensorimotor neuropathy & Rinaldi et al. 2012 \\
Gly262Ser & Slowly progressive mitochondrial encephalomyopathy & Ardissone et al. 2015 \\
Gly308Glu & Early-onset encephalomyopathy & Berger et al. 2011 \\
Leu344Phe & Auditory neuropathy & Zong et al. 2015 \\
Thr260Ala & Auditory neuropathy & Zong et al. 2015 \\
Arg422Trp & Auditory neuropathy & Zong et al. 2015 \\
Arg422Gln & Auditory neuropathy & Zong et al. 2015 \\
Arg451Gln & Auditory neuropathy & Zong et al. 2015 \\
\hline
\end{tabular}


in other forms of mitochondrial encephalopathy such as Leigh syndrome. Although peripheral neuropathy is not uncommon in mitochondrial disorders, it could also indicate a further role for AIFM1 within the nervous system. Alternative explanations for the peripheral neuropathy could include an adverse effect of dichloroacetate. Skeletal muscle findings demonstrated expected findings in mitochondrial disease, including structural abnormalities of the mitochondria. The subsarcolemmal accumulations of glycogen and slightly prominent lipid stores that were present at autopsy, but not in the initial sample from 8 wk of age, were also consistent with progressive mitochondrial dysfunction.

Significant findings were present throughout general examination that could relate directly to AIFM1 mutation. The patient's thymus gland was markedly larger than expected. The postmortem observed weight of $39 \mathrm{~g}$ was substantially larger than the 9.5-g expected weight for an infant of his length (Coppoletta and Wolbach 1933), despite the history of neonatal stress, which typically results in a smaller-than-expected thymus gland. The tingible-body macrophages that are typically associated with apoptosis and thymic regression were lacking. It is interesting to speculate whether the abnormal AIFM1 interfered with the normal apoptotic regression of the thymic gland. The hepatic finding of microvesicular steatosis is a known consequence of disruption or deficiency of the mitochondrial $\beta$-oxidation process (Fromenty and Pessayre 1997), and in the setting of abnormal AIFM1 may be due to direct impairment of oxidative phosphorylation in hepatocytes.

Two pulmonary findings were of interest in this case. Follicular bronchiolitis in the pediatric population is often seen in the setting of genetic immune dysregulation, and its occurrence in conjunction with AIFM1 mutation in this patient is therefore striking. It is possible that the proliferation of bronchus-associated lymphoid tissue might be driven by factors that interact with AIFM1. It is interesting to note that caspase, which may be modulated by AIFM1, is elevated in bronchoalveolar lavage fluid in children, where it has been correlated with bronchiolitis severity (Laham et al. 2010; Mehta et al. 2014). We thus provide preliminary evidence that genetic variants in AIFM1 may be a member of the growing list of heritable mutations contributing to pediatric follicular bronchitis/bronchiolitis. It is also tempting to relate the patient's pulmonary arterial medial hypertrophy to the deficiency of AIFM1. This pulmonary arterial hypertensive remodeling was likely secondary to critical illness, although AIFM1 could increase the likelihood of developing secondary pulmonary hypertension via interaction with the aldosterone axis (Xiao et al. 2014), which in turn is known to modulate pulmonary arterial hypertensive changes (Maron and Leopold 2015).

To summarize, our report is the first to characterize detailed autopsy findings in lactic acidosis and early-onset, rapidly progressive epilepsy of infancy associated with AIFM1 mutation. The findings expand the clinical and pathologic spectra of disease related to AIFM1 abnormality, and provide further insights into the broad relevance that AIFM1 has in human disease.

\section{METHODS}

\section{Genomic Analysis}

\section{Next-Generation Exome Sequencing}

Whole-exome sequencing was completed for the patient and mother. All studies were completed according to approval of the Institutional Review Board of Boston Children's Hospital. Total genomic DNA was extracted from peripheral blood lymphocytes using QIAmp DNA Mini Kit (QIAGEN). DNA from the proband and mother was sent for wholeexome sequencing (WES) at Claritas Genomics. Samples were prepared as an Illumina 
COLD SPRING HARBOR Molecular Case Studies
Fatal encephalomyopathy with AIFM1 mutation sequencing library and enriched for exomic sequences using the Agilent V5 Sureselect kit. The captured libraries were sequenced using Illumina HiSeq 2000 Sequencers at Lab Corp.

\section{Data Analysis}

FASTQs generated from exome sequencing were filtered, aligned, and variants were filtered and annotated by Codified Genomics (proprietary algorithm).

\section{Structural Modeling}

The structural modeling of AIFM1 GIn479Arg and the structural elucidation of both wild-type and mutant AIFM1 were generated using PyMOL (The PyMOL Molecular Graphics System, Version 1.8 Schrödinger, LLC.).

\section{Clinical Record Review}

Medical data were abstracted from electronic records. Growth parameters were calculated using the Centers for Disease Control and Prevention (CDC) data.

\section{Pathologic Examination Muscle Biopsy}

An antemortem open biopsy of quadriceps muscle and a postmortem sampling of psoas muscle were obtained. Muscle was snap frozen in isopentane, cooled in liquid nitrogen, and mounted in optimal cutting temperature (OCT) compound. Six micron sections were cut and mounted onto microscope slides. Gomori trichrome, NADH, ATPase pH 4.6, and COX staining were carried out according to standard protocols. Microscopic sections including $H \& E$, and selected section stained with luxol fast blue (LFB)/Nissl, Bielschowski silver stain, and immunostains (GFAP, NeuN, Factor VIII, neurofilament protein, SMI 31) were examined. A portion of muscle was fixed in gluteraldehyde, embedded in plastic; semithin sections were stained with toluidine blue, and ultrastructural examination was conducted.

\section{Postmortem Examination}

Parents gave consent for a full, unrestricted autopsy. External examination was conducted. Evisceration was conducted through a standard Y-shaped thoracoabdominal incision, and the brain was removed via the calvarium. Organs were examined and dissected in the fresh state, except for the brain, which was fixed before dissection. Histologic sections were fixed in formalin, embedded in paraffin, and sectioned at 5 microns for microscopic examination. Peripheral nerve and skeletal muscle were processed for electron microscopic examination.

\section{ADDITIONAL INFORMATION}

\section{Data Deposition and Access}

Genetic variant data was submitted to ClinVar (https://www.ncbi.nlm.nih.gov/clinvar) under accession number SCV000484975. Raw data were not deposited because of lack of patient consent.

\section{Ethics Statement}

Research protocol was approved by the Institutional Review Board at Boston Children's Hospital. All procedures followed were in accordance with the ethical standards of the responsible committee on human experimentation (institutional and national) and with the 
Competing Interest Statement The authors have declared no competing interest.

Received October 18, 2016; accepted in revised form December 29, 2016
Helsinki Declaration of 1975, as revised in 2000. Oral and written consent for study enrollment and publication was obtained from the subject's parents.

\section{Acknowledgments}

We thank the family for their support and enrollment.

\section{Author Contributions}

All authors were involved in (a) conception and design, or analysis and interpretation of data, and (b) drafting the article or revising it critically for important intellectual content.

\section{Funding}

This work was made possible by grants from the following institutions: P.B.A. was supported by R01 AR068429 from the National Institute of Arthritis and Musculoskeletal and Skeletal Diseases (NIAMS) of the National Institutes of Health (NIH) and U19 HD077671 from the National Institute of Child Health and Human Development (NICHD)/National Human Genome Research Institute (NHGRI)/NIH. The Gene Discovery Core of The Manton Center for Orphan Disease Research, Boston Children's Hospital also supported the work. Sanger sequencing was performed by the Molecular Genetics Core Facility of the Intellectual and Developmental Disabilities Research Center (IDDRC) at Boston Children's Hospital, supported by NIH grant P30 HD18655.

\section{REFERENCES}

Ardissone A, Piscosquito G, Legati A, Langella T, Lamantea E, Garavaglia B, Salsano E, Farina L, Moroni I, Pareyson D, et al. 2015. A slowly progressive mitochondrial encephalomyopathy widens the spectrum of AIFM1 disorders. Neurology 84: 2193-2195.

Berger I, Ben-Neriah Z, Dor-Wolman T, Shaag A, Saada A, Zenvirt S, Raas-Rothschild A, Nadjari M, Kaestner KH, Elpeleg O. 2011. Early prenatal ventriculomegaly due to an AIFM1 mutation identified by linkage analysis and whole exome sequencing. Mol Genet Metab 104: 517-520.

Coppoletta JM, Wolbach SB. 1933. Body length and organ weights of infants and children: a study of the body length and normal weights of the more important vital organs of the body between birth and twelve years of age. Am J Pathol 9: 55-70.

Cowchock FS, Duckett SW, Streletz LJ, Graziani LJ, Jackson LG. 1985. X-linked motor-sensory neuropathy type-Il with deafness and mental retardation: a new disorder. Am J Med Genet 20: 307-315.

Diodato D, Tasca G, Verrigni D, D’Amico A, Rizza T, Tozzi G, Martinelli D, Verardo M, Invernizzi F, Nasca A, et al. 2015. A novel AIFM1 mutation expands the phenotype to an infantile motor neuron disease. Eur J Hum Genet 24: 463-466.

El-Hattab AW, Scaglia F. 2013. Mitochondrial DNA depletion syndromes: review and updates of genetic basis, manifestations, and therapeutic options. Neurotherapeutics 10: 186-198.

Ferreira P, Villanueva R, Martínez-Júlvez M, Herguedas B, Marcuello C, Fernandez-Silva P, Cabon L, Hermoso JA, Lostao A, Susin SA, et al. 2014. Structural insights into the coenzyme mediated monomerdimer transition of the pro-apoptotic apoptosis inducing factor. Biochemistry 53: 4204-4215.

Fromenty B, Pessayre D. 1997. Impaired mitochondrial function in microvesicular steatosis. Effects of drugs, ethanol, hormones and cytokines. J Hepatol 26(Suppl 2): 43-53.

Ghezzi D, Sevrioukova I, Invernizzi F, Lamperti C, Mora M, D’Adamo P, Novara F, Zuffardi O, Uziel G, Zeviani M. 2010. Severe $X$-linked mitochondrial encephalomyopathy associated with a mutation in apoptosis-inducing factor. Am J Hum Genet 86: 639-649.

Joza N, Oudit GY, Brown D, Bénit P, Kassiri Z, Vahsen N, Benoit L, Patel MM, Nowikovsky K, Vassault A, et al. 2005. Muscle-specific loss of apoptosis-inducing factor leads to mitochondrial dysfunction, skeletal muscle atrophy, and dilated cardiomyopathy. Mol Cell Biol 25: 10261-10272.

Klein JA, Longo-Guess CM, Rossmann MP, Seburn KL, Hurd RE, Frankel WN, Bronson RT, Ackerman SL. 2002. The harlequin mouse mutation downregulates apoptosis-inducing factor. Nature 419: 367-374.

Laham FR, Trott AA, Bennett BL, Kozinetz CA, Jewell AM, Garofalo RP, Piedra PA. 2010. LDH concentration in nasal-wash fluid as a biochemical predictor of bronchiolitis severity. Pediatrics 125: e225-e233. 
Maron BA, Leopold JA. 2015. Emerging concepts in the molecular basis of pulmonary arterial hypertension: part II: neurohormonal signaling contributes to the pulmonary vascular and right ventricular pathophenotype of pulmonary arterial hypertension. Circulation 131: 2079-2091.

Mehta R, Scheffler M, Tapia L, Aideyan L, Patel KD, Jewell AM, Avadhanula V, Mei M, Garofalo RP, Piedra PA. 2014. Lactate dehydrogenase and caspase activity in nasopharyngeal secretions are predictors of bronchiolitis severity. Influenza Other Respir Viruses 8: 617-625.

Miramar MD, Costantini P, Ravagnan L, Saraiva LM, Haouzi D, Brothers G, Penninger JM, Peleato ML, Kroemer G, Susin SA. 2001. NADH oxidase activity of mitochondrial apoptosis-inducing factor. J Biol Chem 276: 16391-16398.

Møller RS, Jensen LR, Maas SM, Filmus J, Capurro M, Hansen C, Marcelis CLM, Ravn K, Andrieux J, Mathieu M, et al. 2014. X-linked congenital ptosis and associated intellectual disability, short stature, microcephaly, cleft palate, digital and genital abnormalities define novel Xq25q26 duplication syndrome. Hum Genet 133: 625-638.

Pospisilik JA, Knauf C, Joza N, Benit P, Orthofer M, Cani PD, Ebersberger I, Nakashima T, Sarao R, Neely G, et al. 2007. Targeted deletion of AIF decreases mitochondrial oxidative phosphorylation and protects from obesity and diabetes. Cell 131: 476-491.

Rinaldi C, Grunseich C, Sevrioukova IF, Schindler A, Horkayne-Szakaly I, Lamperti C, Landouré G, Kennerson ML, Burnett BG, Bönnemann C, et al. 2012. Cowchock syndrome is associated with a mutation in apoptosis-inducing factor. Am J Hum Genet 91: 1095-1102.

Sevrioukova IF. 2016. Structure/function relations in AIFM1 variants associated with neurodegenerative disorders. J Mol Biol 428: 3650-3665.

Susin SA, Lorenzo HK, Zamzami N, Marzo I, Snow BE, Brothers GM, Mangion J, Jacotot E, Costantini P, Loeffler M, et al. 1999. Molecular characterization of mitochondrial apoptosis-inducing factor. Nature 397: 441-446.

Tang S, Wang J, Lee N-C, Milone M, Halberg MC, Schmitt ES, Craigen WJ, Zhang W, Wong L-JC. 2011. Mitochondrial DNA polymerase $\gamma$ mutations: an ever expanding molecular and clinical spectrum. $J$ Med Genet 48: 669-681.

Vahsen N, Candé C, Brière J-J, Bénit P, Joza N, Larochette N, Mastroberardino PG, Pequignot MO, Casares N, Lazar V, et al. 2004. AlF deficiency compromises oxidative phosphorylation. EMBO J 23: 4679-4689.

Wang QJ, Li QZ, Rao SQ, Lee K, Huang XS, Yang WY, Zhai SQ, Guo WW, Guo YF, Yu N, et al. 2006. AUNX1, a novel locus responsible for $X$ linked recessive auditory and peripheral neuropathy, maps to $\mathrm{Xq23-27.3.} J$ Med Genet 43: e33.

Wong L-JC. 2012. Mitochondrial syndromes with leukoencephalopathies. Semin Neurol 32: 55-61.

Xiao T-T, Wang Y-Y, Zhang Y, Bai C-H, Shen X-C. 2014. Similar to spironolactone, oxymatrine is protective in aldosterone-induced cardiomyocyte injury via inhibition of calpain and apoptosis-inducing factor signaling. PLoS One 9: e88856.

Zimoń M, Baets J, Almeida-Souza L, De Vriendt E, Nikodinovic J, Parman Y, Battaloğlu E, Matur Z, Guergueltcheva V, Tournev I, et al. 2012. Loss-of-function mutations in HINT1 cause axonal neuropathy with neuromyotonia. Nat Genet 44: 1080-1083.

Zong L, Guan J, Ealy M, Zhang Q, Wang D, Wang H, Zhao Y, Shen Z, Campbell CA, Wang F, et al. 2015. Mutations in apoptosis-inducing factor cause $X$-linked recessive auditory neuropathy spectrum disorder. J Med Genet 52: 523-531. 


\section{COLD SPRING HARBOR Molecular Case Studies}

\section{AIFM1 mutation presenting with fatal encephalomyopathy and mitochondrial disease in an infant}

Sarah U. Morton, Sanjay P. Prabhu, Hart G.W. Lidov, et al.

Cold Spring Harb Mol Case Stud 2017, 3: a001560 originally published online January 10, 2017 Access the most recent version at doi: $10.1101 / \mathrm{mcs} . a 001560$

References This article cites 27 articles, 7 of which can be accessed free at: http://molecularcasestudies.cshlp.org/content/3/2/a001560.full.html\#ref-list-1

License This article is distributed under the terms of the Creative Commons Attribution-NonCommercial License, which permits reuse and redistribution, except for commercial purposes, provided that the original author and source are credited.

Email Alerting Receive free email alerts when new articles cite this article - sign up in the box at the Service top right corner of the article or click here. 